\title{
Flux- and gradient-driven global gyrokinetic simulation of tokamak turbulence
}

Tobias Görler, ${ }^{1, \text { a) }}$ Xavier Lapillonne, ${ }^{2}$ Stephan Brunner, ${ }^{3}$ Tilman Dannert, ${ }^{3}$ Frank

Jenko, ${ }^{3}$ Sohrab Khosh Aghdam, ${ }^{3}$ Patrick Marcus, ${ }^{3}$ Ben F McMillan, ${ }^{3}$ Florian Merz, ${ }^{3}$

Olivier Sauter, ${ }^{3}$ Daniel Told, ${ }^{3}$ and Laurent Villard ${ }^{3}$

1) Max-Planck-Institut für Plasmaphysik, IPP-EURATOM Association, Garching, Germany

2) École Polytechnique Fédérale de Lausanne, Centre de Recherches en Physique des Plasmas, Association Euratom-Confédération Suisse, CH-1015 Lausanne, Switzerland

3) Rechenzentrum der Max-Planck-Gesellschaft und des Max-PlanckInstitutes für Plasmaphysik, EURATOM Association, D-85748, Germany

The Eulerian gyrokinetic turbulence code GENE has recently been extended to a full torus code. Moreover, it now provides Krook-type sources for gradient-driven simulations where the profiles are maintained on average as well as localized heat sources for a flux-driven type of operation. Careful verification studies and benchmarks are performed successfully. This setup is applied to address three related transport issues concerning nonlocal effects. First, it is confirmed that in gradient-driven simulations, the local limit can be reproduced - provided that finite aspect ratio effects in the geometry are treated carefully. In this context, it also becomes clear that the profile widths (not the device width) may constitute a more appropriate measure for finite size effects. Second, the nature and role of heat flux avalanches are discussed in the framework of both local and global, flux- and gradient-driven simulations. Third, simulations dedicated to discharges with electron internal barriers are addressed.

a)Electronic mail: tobias.goerler@ipp.mpg.de 


\section{INTRODUCTION}

One of the remaining key physics problems on the way to efficient fusion power plants based on toroidal magnetic confinement is the thorough understanding and reliable prediction of the so-called anomalous transport of heat, momentum, and particles across the magnetic surfaces (hereafter referred to as the radial direction). It is by now commonly attributed to small-scale (roughly comparable to the ion or electron gyroradius), low-frequency (much smaller than the ion and electron gyrofrequency) turbulence which is driven by various plasma microinstabilities where the latter extract free energy from the background temperature and density gradients. An appropriate theoretical framework for such hightemperature, low-density and thus weakly collisional plasmas - as they occur in magnetic confinement fusion - is provided by the gyrokinetic approach ${ }^{1,2}$ where fast dynamics (e.g., the particle gyromotion) are eliminated from the full kinetic description but low-frequency physics is kept. However, the resulting gyrokinetic Vlasov-Maxwell system of equations in the five-dimensional phase space is generally way too complicated to be solved analytically. A number of dedicated numerical tools have thus been developed which can, e.g. be classified into so-called local and global codes. The former are also called flux-tube ${ }^{3-5}$ codes since they consider just a narrow box size perpendicular to a magnetic field line. Consequently, temperature and density profiles and their gradients are only evaluated at the (radial) center position of this domain and periodic boundary conditions allowing for the application of fast and efficient spectral methods are employed. In addition, a so-called $\delta f$ splitting is applied, i.e. the fluctuating part $f_{1}$ of the distribution function is considered to be small compared to the stationary background part $f_{0}$. In this case, however, one implicitly assumes a gyroBohm transport scaling, i.e. a Bohm scaling reduced by the gyroradius-to-machine-size ratio $\rho^{*}$ where the latter has to be small. In order to determine the limit of such a-priori scalings

and for applications to small fusion devices or peaked gradient profiles one thus has to rely on global codes where full radial temperature, density and geometry profiles are considered. Amongst others, an implementation of both approaches can be found in the software package $\mathrm{GENE}^{6-9}$ which is a massively parallelized, comprehensive Eulerian $\delta f$ code. In this paper, we present comparisons of the recently developed global version with the wellestablished local code version and the global gyrokinetic Lagrangian PIC code ORB5 $5^{10,11}$ and hereby study the role of finite-size effects. One specific presumably nonlocal effect, 
so-called heat flux avalanches, is picked for a more detailed investigation and discussion. Furthermore, first results for parameters being extracted from a TCV tokamak discharge with an electron-Internal Transport Barrier are presented.

\section{A BRIEF INTRODUCTION TO GENE}

As most results presented in this contribution are based on the software package GENE, a brief code introduction shall be given in the present section. A more detailed description of the global version of GENE can be found in Refs. 9,12, and 13.

The employed theoretical framework is the gyrokinetic theory. Here, a gyrocenter distribution function $f_{\sigma}$ per species $\sigma$ with mass $m_{\sigma}$ and charge $q_{\sigma}$ is evolved in time using an accordingly transformed Vlasov equation (or Boltzmann equation if weak collisions are considered) which reads in advection form (see, for instance, Ref. 1)

$$
\frac{\partial f_{\sigma}}{\partial t}+\dot{\mathbf{X}} \cdot \nabla f_{\sigma}+\dot{v}_{\|} \frac{\partial f_{\sigma}}{\partial v_{\|}}+\dot{\mu} \frac{\partial f_{\sigma}}{\partial \mu}=0
$$

where the magnetic moment is an adiabatic invariant fulfilling $\dot{\mu}=0$. In the low- $\beta$ limit i.e., the thermal pressure is small compared to the magnetic pressure - the time derivatives of the gyrocenter coordinate $\mathbf{X}$ and the parallel velocity $v_{\|}$are given by

$$
\dot{\mathbf{X}}=v_{\|} \hat{\mathbf{b}}_{0}+\frac{B_{0}}{B_{0 \|}^{*}}\left(\mathbf{v}_{\bar{\chi}}+\mathbf{v}_{\nabla B}+\mathbf{v}_{c}\right) \quad \text { and } \quad \dot{v}_{\|}=-\frac{\dot{\mathbf{X}}}{m_{\sigma} v_{\|}} \cdot\left(q_{\sigma} \nabla \bar{\phi}_{1}+\frac{q_{\sigma}}{c} \hat{\mathbf{b}}_{0} \dot{\bar{A}}_{1 \|}+\mu \nabla B_{0}\right) .
$$

In these equations and in the following, $B_{0}$ denotes the modulus of the magnetic (background) field vector $\mathbf{B}_{0}, \hat{\mathbf{b}}_{0}=\mathbf{B}_{0} / B_{0}$ the corresponding unit vector, $B_{0 \|}^{*}=\hat{\mathbf{b}}_{0} \cdot \mathbf{B}_{0}^{*}$ the parallel component of $\mathbf{B}_{0}^{*}=\nabla \times\left(\mathbf{A}_{0}+\frac{m_{\sigma} c}{q_{\sigma}} v_{\|} \hat{\mathbf{b}}_{0}\right)$, and $\bar{\chi}_{1}=\bar{\phi}_{1}-\frac{v_{\|}}{c} \bar{A}_{1 \|}$ the gyroaveraged scalar potential in the gyrocenter moving frame with the fluctuating fractions of the electrostatic potential $\phi_{1}$ and the parallel vector field component $A_{1 \|}$. The total drift velocity consists of the generalized $\mathbf{E} \times \mathbf{B}$ velocity $\mathbf{v}_{\bar{\chi}}=\frac{c}{B_{0}} \hat{\mathbf{b}}_{0} \times \nabla \bar{\chi}_{1}$, the gradient-B velocity $\mathbf{v}_{\nabla B_{0}}=\frac{\mu c}{q_{\sigma} B_{0}} \hat{\mathbf{b}}_{0} \times \nabla B_{0}$, and the curvature drift velocity $\mathbf{v}_{c}=\frac{m_{\sigma} c}{q_{\sigma} B_{0}} v_{\|}^{2} \hat{\mathbf{b}}_{0} \times \frac{\nabla B_{0}}{B_{0}}$. Overbars and $\langle\ldots\rangle$ brackets denote gyroaverages being defined as $\bar{\phi}_{1}(\mathbf{X}) \equiv \mathcal{G}\left[\phi_{1}(\mathbf{X})\right] \equiv \frac{1}{2 \pi} \oint \mathrm{d} \theta \phi_{1}(\mathbf{X}+\mathbf{r}(\theta))$ with the gyroradius vector $\mathbf{r}(\theta)$ being orthogonal to the magnetic field.

Since observables like density and temperature often tend to exhibit fluctuating parts being much smaller than the quasi-stationary mean values, the aforementioned $\delta f$ splitting is furthermore applied, as well. With the latter being chosen here as a local Maxwellian, the 
gyrokinetic Vlasov equation can be rewritten and simplified. Keeping afterwards just first order terms in the perturbation parameter expansion, the $\nabla \chi \times \mathbf{B}$ nonlinearity is retained while higher-order terms like the so-called $v_{\|}$-nonlinearity are neglected. This is in line with careful studies in Refs. 14-16.

The distribution functions for the different species are coupled in the low- $\beta$ approximation through the gyrokinetic Poisson equation and the parallel component of Ampère's law. The former reads

$$
-\nabla_{\perp}^{2} \phi_{1}=4 \pi \sum_{\sigma}\left(q_{\sigma} \bar{n}_{1 \sigma}+n_{0 \sigma} \frac{q_{\sigma}^{2}}{T_{0 \sigma}}\left[\frac{B_{0}}{T_{0 \sigma}} \int\left\langle\bar{\phi}_{1}(\mathbf{x}-\mathbf{r})\right\rangle \mathrm{e}^{-\frac{\mu B_{0}}{T_{0 \sigma}}} \mathrm{d} \mu-\phi_{1}(\mathbf{x})\right]\right)
$$

where the gyrocenter density is $\bar{n}_{1 \sigma}=\frac{2 \pi}{m_{\sigma}} \iint B_{0 \|}^{*}\left\langle f_{1 \sigma}(\mathbf{x}-\mathbf{r})\right\rangle \mathrm{d} v_{\|} \mathrm{d} \mu$. If adiabatic electrons are assumed, the electron contribution on the right hand side of Eq. (2) is replaced by $n_{1 e}=\frac{e n_{0 e}}{T_{0 e}}\left(\phi_{1}-\left\langle\phi_{1}\right\rangle_{\mathrm{FS}}\right)$, with $\langle\ldots\rangle_{\mathrm{FS}}$ indicating a flux surface average. Note, that derivatives of equilibrium quantities are here ordered small (as before). The second field equation is

$$
-\nabla_{\perp}^{2} A_{1 \|}=\frac{4 \pi^{2} B_{0}}{c} \sum_{\sigma} q_{\sigma} \iint\left\langle f_{1 \sigma}(\mathbf{x}-\mathbf{r})\right\rangle v_{\|} \mathrm{d} v_{\|} \mathrm{d} \mu .
$$

The gyrokinetic Vlasov-Maxwell system of equations, Eqs. (1)-(3), is evaluated in GEnE in the first-order perturbation expansion using a field-aligned coordinate system. The latter allows to take advantage of the strong anisotropy of plasma turbulent transport and thus use just a few (on the order of several tens) grid points in the direction along the field line where turbulent structures hardly vary. This concept is quite similar to a flux tube in local codes and indeed uses comparable quasi-periodic boundary conditions in the parallel $(z)$ direction which account for the stretching of the simulation box due to magnetic shear. However, in contrast to a flux tube which is very narrow in both directions $(x, y)$ perpendicular to the magnetic field, the simulation domain in the global code can be the full torus or at least a wedge which encompasses a flux bundle. Periodic boundary conditions are hence inapplicable since full radial profiles, e.g. of temperatures and densities have to be considered in this case. Consequently, (pseudo-) spectral methods can only be employed in the binormal ( $y$ ) direction which exploits the axisymmetry. One major consequence is that gyroaverage operators, for instance, cannot be given a simple analytic form as in the $\left(k_{x}, k_{y}\right)$ Fourier space. Hence, the integration over gyroangles has to be performed using interpolation techniques in real space for the radial direction. In GENE, the latter is realized using finite element interpolation which effectively amounts to a Hermite polynomial interpolation, for details see Ref. 9. 
Besides delocalized hyperdiffusion terms which are able to compensate for numerical artifacts when representing derivatives by dissipation-less finite differencing schemes (see Ref. 17), the following source and sink terms can be activated in GENE. First, a simple Krook operator, $\mathrm{d} f_{1 \sigma} / \mathrm{d} t=-S_{\text {Krook }}(x) f_{1 \sigma}$, can be applied with vanishing amplitude in the inner part of the simulation domain in order to create artificial buffer zones where fluctuations are damped to be consistent with fixed boundary conditions.

For gradient-driven simulations, a Krook-type heat source term similar to the model in Ref. 11,

$\mathcal{S}_{K H}\left(x,\left|v_{\|}\right|, \mu\right)=-\gamma_{h}\left[\left\langle f_{1 \sigma}\left(\mathbf{X},\left|v_{\|}\right|, \mu\right)\right\rangle_{\mathrm{FS}}-\left\langle f_{0 \sigma}\left(\mathbf{X},\left|v_{\|}\right|, \mu\right)\right\rangle_{\mathrm{FS}} \frac{\left\langle\int d \mathbf{v}\left\langle f_{1 \sigma}\left(\mathbf{X},\left|v_{\|}\right|, \mu\right)\right\rangle_{\mathrm{FS}}\right\rangle_{\mathrm{FS}}}{\left\langle\int d \mathbf{v}\left\langle f_{0 \sigma}\left(\mathbf{X}, v_{\|}, \mu\right)\right\rangle_{\mathrm{FS}}\right\rangle_{\mathrm{FS}}}\right]$,

with $f_{1 \sigma}\left(\mathbf{X},\left|v_{\|}\right|, \mu\right)=\left(f_{1 \sigma}\left(\mathbf{X}, v_{\|}, \mu\right)+f_{1 \sigma}\left(\mathbf{X},-v_{\|}, \mu\right)\right) / 2$, is available which can be added to the right hand side of the Vlasov equation. Being applied over the whole radial simulation domain, it is designed to fix the temperature profile on average, while leaving the fluxsurface averaged density and parallel momentum unaffected. If density profiles shall be approximately maintained as well, the operator

$$
\mathcal{S}_{K P}\left(x,\left|v_{\|}\right|, \mu\right)=-\gamma_{p}\left\langle\int d \mathbf{v}\left\langle f_{1 \sigma}\left(\mathbf{X},\left|v_{\|}\right|, \mu\right)\right\rangle_{\mathrm{FS}}\right\rangle_{\mathrm{FS}} \frac{\left\langle f_{0 \sigma}\left(\mathbf{X},\left|v_{\|}\right|, \mu\right)\right\rangle_{\mathrm{FS}}}{\left\langle\int d \mathbf{v}\left\langle f_{0 \sigma}\left(\mathbf{X}, v_{\|}, \mu\right)\right\rangle_{\mathrm{FS}}\right\rangle_{\mathrm{FS}}}
$$

can be used.

If a flux-driven type of operation is desired where the profiles are explicitly allowed to adjust to a prescribed heat source, an implementation of a localized heat source model closely following Ref. 18 is at hand. In normalized units, it is added to the right hand side of the Vlasov equation as $\frac{\mathrm{d} f_{1}}{\mathrm{~d} t}=\mathcal{S}_{H}=\mathcal{S}_{0} \mathcal{S}_{x} \mathcal{S}_{E}$ with

$$
\mathcal{S}_{E}=\frac{2}{3} \frac{1}{p_{0 \sigma}(x)}\left(\frac{v_{\|}^{2}+\mu B_{0}}{T_{0 \sigma}(x) / T_{0 \sigma}\left(x_{0}\right)}-\frac{3}{2}\right) f_{0 \sigma}, \quad \mathcal{S}_{x}=\mathcal{S}_{x, i n}(x) / \int \mathrm{d}^{3} x \mathcal{S}_{x, i n}(x) J(x, z),
$$

and the source amplitude $\mathcal{S}_{0}$. Here, $J(x, z)$ denotes the configuration space Jacobian and $\mathcal{S}_{x, i n}(x)$ is a user-defined radial source profile which is, for instance, Gaussian-like.

Detailed verification studies and benchmarks of the global GENE extension as, for instance, Rosenbluth-Hinton test results, intercode comparisons with the linear global gyrokinetic particle-in-cell (PIC) code GYGLES ${ }^{19}$ and the global gyrokinetic PIC code OrB5 can be found in Refs. 9 and 20. 


\section{FINITE SIZE EFFECTS AND CONVERGENCE TOWARDS THE LOCAL LIMIT}

\section{A. Linear GENE results}

An important and yet not fully answered question arising in plasma microturbulence studies is the possible influence of finite-size effects on the turbulence itself. For instance, if the turbulent eddies would tend to be comparable to the machine size, they would obviously feel the limiting boundaries. Given a more realistic situation, one might consider turbulent mode structures which cover at least a significant fraction of the radial profiles of temperature, density and the magnetic topology and thus exhibit nonlocal behavior. The most popular parameter in this context is the gyroradius-to-machine-size ratio $\rho^{*}$ which is almost always set to $\rho^{*}=\rho_{i} / a$ (with ion gyroradius $\rho_{i}$ ) as the gradient length scales are roughly on the same order as the Tokamak minor radius $a$. Clearly, the limit $\rho^{*} \rightarrow 0$ describes a situation where turbulent vortices (being on the order of a gyroradius $\rho_{i}$ ) are just susceptible to the gradient drive in an infinitesimal small, i.e. local, domain. Global and local codes should hence agree in this limit which can be used to, e.g. check the implementation of the different numerical schemes. The quantity of interest is, however, the degree of convergence towards the local results for given profiles as $\rho^{*}$ is decreased.

First insights can be derived from linear simulations when displaying the growth rate as function of $\rho^{*}$. Corresponding GENE results are presented in the following. Here, the temperature and density profile shapes for the global simulations are either chosen to exhibit peaked logarithmic gradient profiles by considering

$$
\left(T_{\sigma}, n_{\sigma}\right)=\left(T_{\mathrm{ref}}, n_{\mathrm{ref}}\right) \exp \left[-\kappa_{\left(T_{\sigma}, n_{\sigma}\right)} \varepsilon \Delta(T, n) \tanh \left(\frac{\left(x-x_{0}\right) / a}{\Delta(T, n)}\right)\right],
$$

or flat top gradient profiles by choosing

$$
\left(T_{\sigma}, n_{\sigma}\right)=\left(T_{\text {ref }}, n_{\text {ref }}\right)\left[\frac{\cosh \left(\frac{\left(x-x_{0}\right) / a+\Delta(T, n)}{\delta(T, n)}\right)}{\cosh \left(\frac{\left(x-x_{0}\right) / a-\Delta(T, n)}{\delta(T, n)}\right)}\right]^{-\kappa_{\left(T_{\sigma}, n_{\sigma}\right)} \varepsilon \delta(T, n) / 2}
$$

In definitions (7) and (8), $T_{\text {ref }}$ and $n_{\text {ref }}$ denote reference temperatures and densities at the center position $x_{0}$ (here, $\left.x_{0}=0.5 a\right)$. Furthermore, $\delta(T, n)$ and $\Delta(T, n)$ are characteristic gradient profile widths being set to $\Delta T=\Delta n=(\mathrm{A}) 0.3$, (B) 0.2 , (C) 0.1, (D) 0.2, (E) 0.15 and $(\mathrm{F}) 0.1$, see Fig. 1. The remaining parameters are $\kappa_{T}=\max \left(R_{0} / L_{T}\right)$ and $\kappa_{n}=$ 

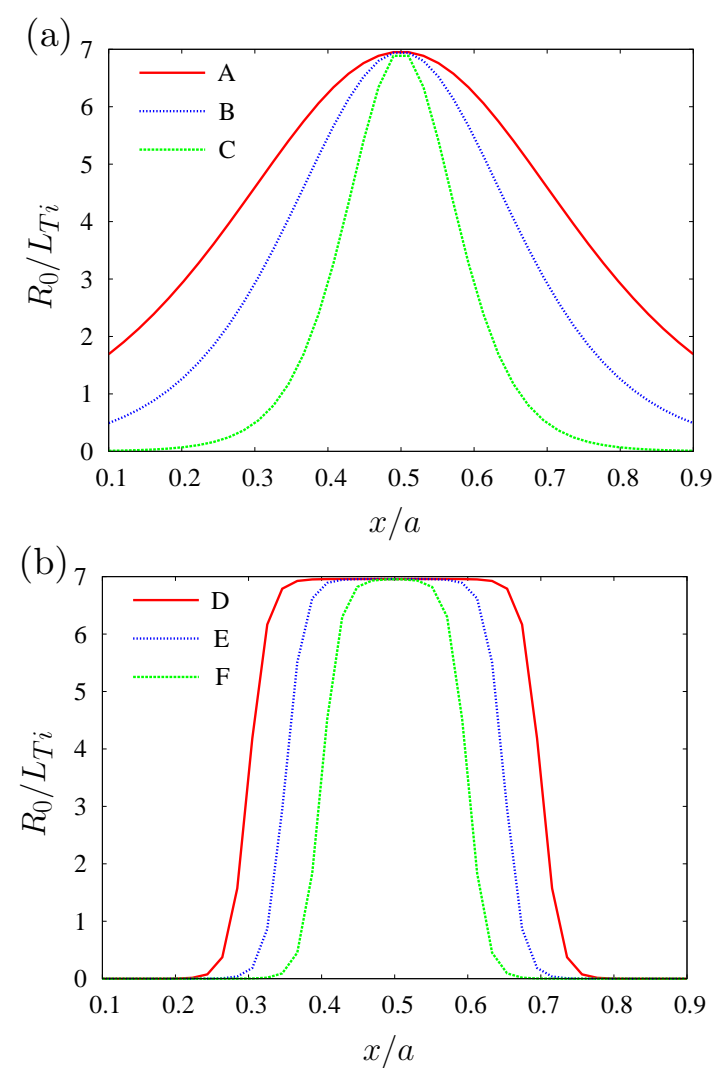

FIG. 1. (Color online) The (a) peaked and (b) flat logarithmic temperature gradient profile for different settings of $\Delta T, \Delta n=(\mathrm{A}) 0.3$, (B) 0.2, (C) 0.1, (D) 0.2, (E) 0.15 and (F) 0.1 .

$\max \left(R_{0} / L_{n}\right)$ which denote the maximum temperature and density gradient values and $\varepsilon$ the inverse aspect ratio between minor radius $a$ and major radius $R_{0}$. Unless stated otherwise, these values will be chosen as in the Cyclone Base Case $(\mathrm{CBC})^{21}$ parameter set, i.e. $\kappa_{T}=$ 6.96, $\kappa_{n}=2.23$ and $\varepsilon=0.36$. The shape of the flux surfaces is assumed to be circular and concentric such that $x$ can be identified with the minor radius $r$ of each flux surface (see Ref. 22 for details). The safety factor profile is $q(x / a)=0.498(x / a)^{4}-0.466(x / a)^{3}+$ $2.373(x / a)^{2}+0.854$ where the center values of $q_{0}=q\left(x_{0}\right)=1.42$ and shear $\hat{s}_{0}=\hat{s}\left(x_{0}\right)=$ 0.8 match the CBC values. The radial boundary condition for the distribution function and the fields is chosen to be Dirichlet type, i.e. the values outside the simulation box are set to zero. In a first step, the linear growth rates at $k_{y} \rho_{i} \approx 0.28$ are investigated using kinetic ions and electrons with $m_{i} / m_{e}=1836$ and $\beta_{e}=0$. Obviously, the global results are approaching the local limit with decreasing $\rho^{*}$, see Fig. 2. The exact convergence behavior does, however, depend on the gradient profile shape with broader profiles exhibiting less differences compared to the local growth rate. For possible explanations of this effect (amongst others, based on ballooning representation arguments), the reader is referred to 

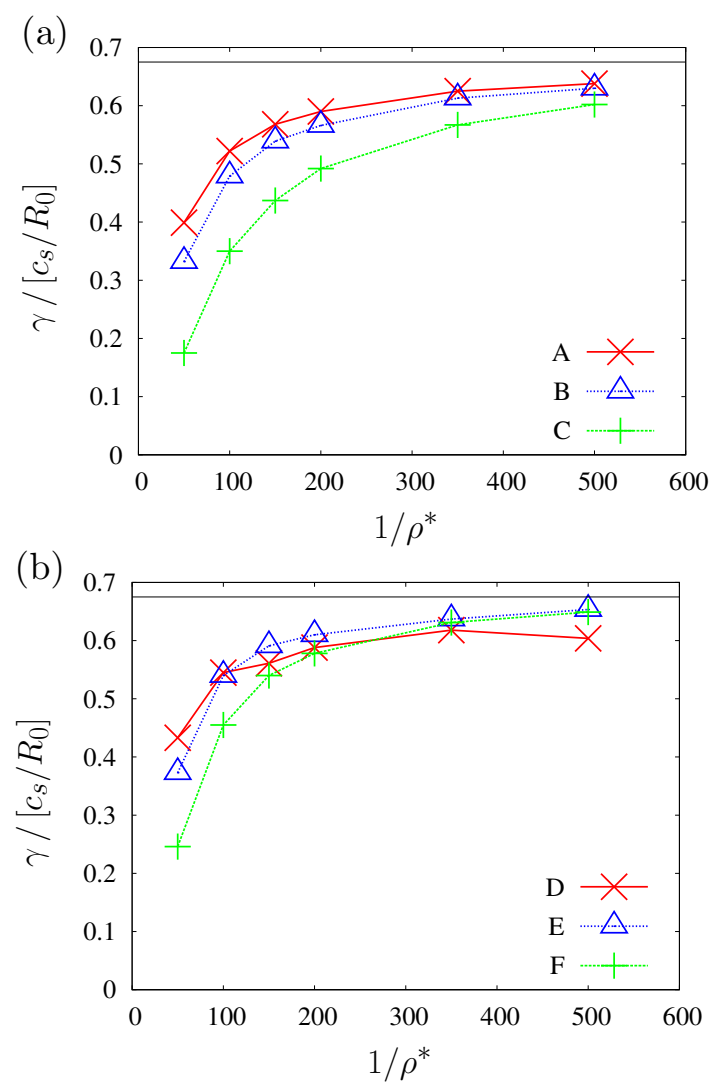

FIG. 2. (Color online) The growth rate vs. $\rho^{*}$ using the (a) peaked and (b) flat logarithmic temperature gradient profile for different settings of $\Delta T$ and $\Delta n$. The local code result is shown as thin, black line.

Ref. 26 and references therein. At very large profile widths - e.g. in case (D) - the modes start to peak at different radial positions thus complicating the analysis and deviating from the general trend. Ignoring these data points, it is possible to cast all remaining growth rate values (see Fig. 3) into a single curve per profile shape by using a new parameter $\rho_{\text {eff }}^{*}=\rho_{i} / \Delta(T, n)$ as has been suggested in Ref. 23. Considering the effective driving region is thus more appropriate than simply taking into account the machine size. One important application at hand are transport barriers, where gradient widths indeed tend to be much smaller than the minor radius.

The convergence towards the local results is not restricted to the aforementioned parameter set but can be observed for a wide range of physical parameters. In the following, further simulation results - namely, an ITG-Kinetic Ballooning (Alfvénic ITG) Mode transition with gyrokinetically treated electrons (with true proton-electron mass ratio) and electromagnetic fluctuations - are shown in Fig. 4. For these runs, the peaked logarithmic 

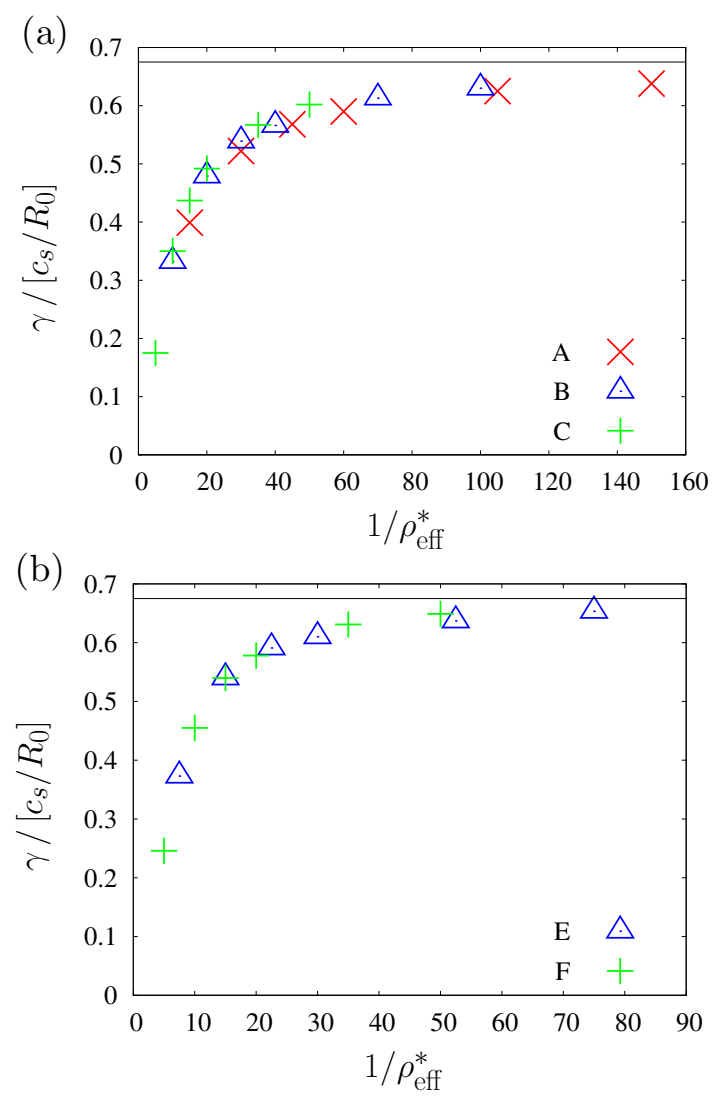

FIG. 3. (Color online) The growth rate vs. $\rho_{\text {eff }}^{*}$ using the (a) peaked and (b) flat logarithmic temperature gradient profile for different settings of $\Delta T$ and $\Delta n$. The broadest flat logarithmic profile is suppressed due to mode peaking.

gradient profile, Eq. (7), has been employed with fixed $\Delta T, \Delta n=0.3$. The left plot still considers $l_{x} / a=$ const. and contains the linear growth rate for different values of $\rho^{*}$ and $\beta_{e}=8 \pi p_{e 0} / B_{\text {ref }}^{2}$ with reference pressure $p_{\text {ref }}$ and magnetic field $B_{\text {ref }}$. Obviously, the previously observed convergence behavior seems to hold even for a wide range of $\beta_{e}$ values since the global growth rates do agree well with the local ones for $\rho^{*} \lesssim 1 / 300$. A comparison with a different kind of $\rho^{*}$ scan is presented in Fig. 4(b) for a fixed $\beta_{e}$ value of $2.5 \%$. Here, the box size is kept fixed with respect to the gyroradius $\rho_{i}$ such that the box size in units of the minor radius is decreasing with $\rho^{*}$. In this case, periodic boundary conditions are employed and a slightly faster convergence towards the local limit can be observed.

With the prediction of heat and particle fluxes being the true motivation for performing gyrokinetic simulations, it is now most interesting to study the nonlinear physics and the underlying transport scaling. Although this issue has already been addressed in the past ${ }^{24,25}$, no coherent picture has emerged so far. In the following, we present results of nonlinear 

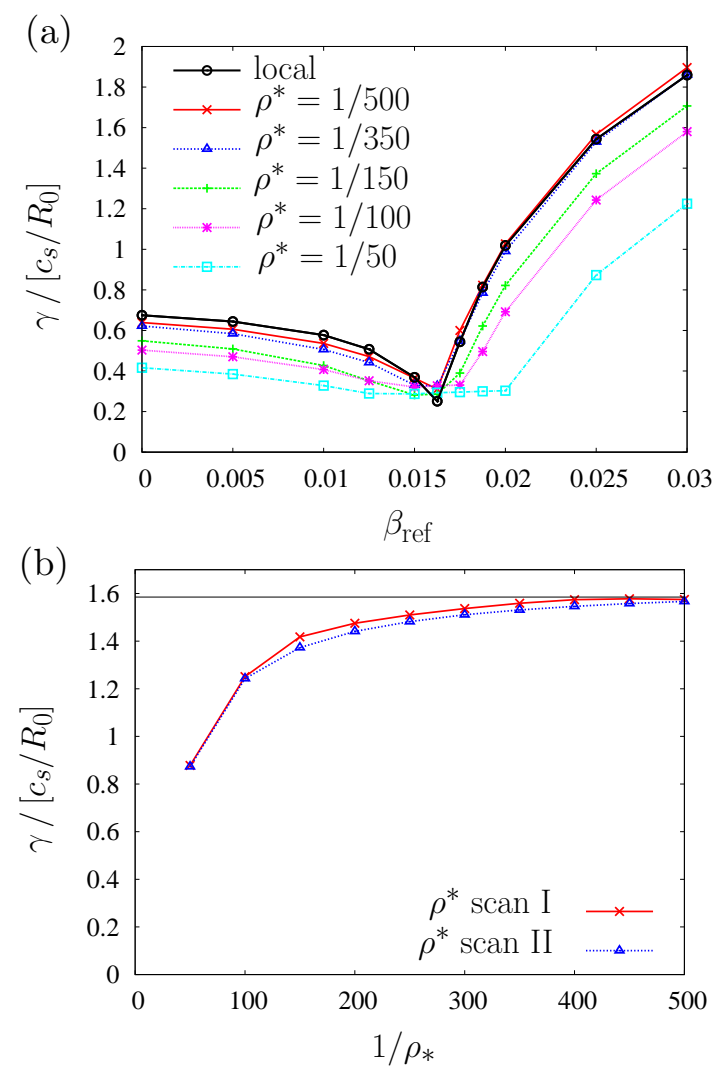

FIG. 4. (Color online) (a) Growth rate at $k_{y} \rho_{i} \approx 0.284$ as function of $\beta_{e}$ and parametrized by different values of $\rho^{*}$ together with the local code result. (b) Growth rate at the same binormal wave number but fixed $\beta_{e}=2.5 \%$ as function of the inverse $\rho^{*}$ value. Here, the radial simulation box is kept fixed with respect to (I) the gyroradius and (II) the minor radius. The local code result using the maximum gradients is again shown as thin, black line. The ideal MHD ballooning mode threshold approximately evaluates to $\beta_{e} \approx 1.6 \%$.

gyrokinetic simulations using adiabatic electrons with $T_{e}=T_{i}$ for different values of $\rho^{*}$ gained from Gene and the Lagrangian PIC code ORB5. Here, the Krook-type heat sources have been applied in order to approximately maintain the average profiles (being similar to the previously used flat logarithmic gradient profile shape)

$$
R_{0} \frac{\mathrm{d} \ln \left(T_{i}, n\right)}{\mathrm{d} x}=\kappa_{\left(T_{i}, n\right)}\left[1-\cosh ^{-2}\left(\frac{x-x_{0}-\delta / 2}{a \Delta}\right)-\cosh ^{-2}\left(\frac{x-x_{0}+\delta / 2}{a \Delta}\right)\right]
$$

taken for $\left|x-x_{0}\right| \leq \delta / 2$ and zero otherwise with $\delta=0.8 a, \Delta=0.04, x_{0}=a / 2$, and the maximum logarithmic gradient lengths $\kappa_{n}=2.2$ and $\kappa_{T_{i}}=7.1$ and 7.5. The latter is indeed set to these two different initial values in order to allow for a linear interpolation of the timeaveraged heat diffusivities at the $\mathrm{CBC}$ value of $\kappa_{T_{i}}=6.96$ in the quasi-stationary saturation phase where the initial temperature profiles are relaxed by some amount but still clearly 
above the nonlinear threshold. Again, the flux surfaces are assumed to be circular concentric with a safety factor profile of $q(x)=0.85-0.01 x / a+2.28(x / a)^{2}-0.09(x / a)^{3}+0.22(x / a)^{4}$. Further details, e.g. on the numerical parameters, can be found in Refs. 20,23,and 26. The resulting $\rho^{*}$ dependencies of the ion heat diffusivity measured in units of $\chi_{\mathrm{gB}}=\rho_{i}^{2} c_{s} / a$ are shown in Fig. 5. First of all, both codes show excellent agreement though they are based on

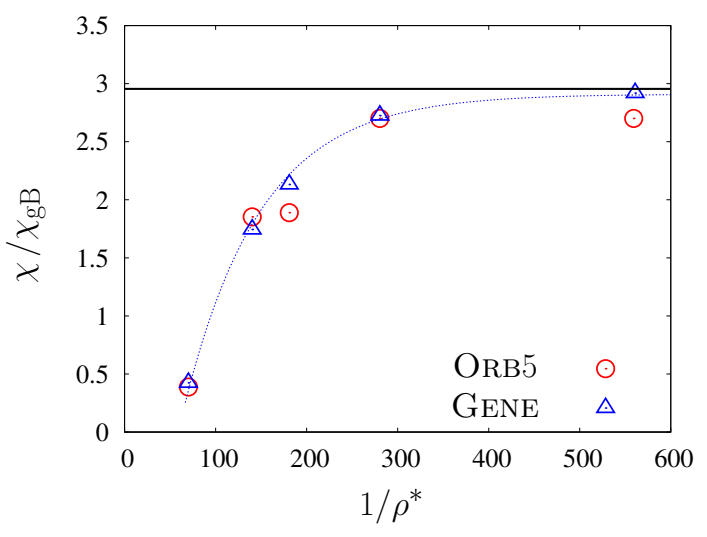

FIG. 5. (Color online) The ORB5 and Gene results are plotted for a series of $\rho^{*}$ values and compared to the ion heat diffusivity of a local GENE simulation being shown as a black line. The function $f(x)=2.91\left(1-2 \mathrm{e}^{-\left(1 / \rho^{*}\right) / 85}\right)$ resulting from a fit to the GENE data points has been added as dashed line.

completely different numerical methods and are thus potentially subject to different types of discretization errors. Within the error bars, which are discussed in Refs. 20 and 23, both codes approach the local GENE (flux tube) result at about $\rho^{*} \lesssim 1 / 500$ such that gyroBohm scaling would hold for large devices as ITER. Comparing with earlier results using similar parameters, the asymptotic value agrees quantitatively with the largest GTC run of Ref. 24. Qualitatively, these results concur with the conclusion of Ref. 25 that the global results converge towards the local results in the $\rho^{*} \rightarrow 0$ limit. The exact value in the latter publication is interestingly quite close to the GENE result using an $\hat{s}-\alpha$ magnetic equilibrium model which differs in the treatment of small inverse aspect ratio terms ${ }^{22}$. Hence, the current investigations imply that small but decisive differences in the equilibrium models are very likely the reason for the earlier disagreement in the $\rho^{*}$ scalings. However, when comparing with experiments, it should be noted that profile shapes, for instance, might have a strong influence on the finite $\rho^{*}$ convergence behavior as has already been observed above in linear investigations and in Refs. 25 and 23. In Ref. 23, it was shown that the transport level for 
adiabatic electron ITG simulations depends on the effective parameter $\rho_{\text {eff }}^{*}$, as well. Thus deviations from gyro-Bohm scaling might occur even in ITER for cases of localized profile gradients such as internal transport barriers.

\section{AVALANCHES IN LOCAL AND GLOBAL SIMULATIONS}

As the anomalous transport scaling appears to be gyro-Bohm-like at small $\rho^{*}$ but nongyro-Bohm-like at $\rho^{*} \gtrsim 1 / 300$, the question as to which mechanisms are responsible for this transition arises. An often favored candidate are so-called avalanches, i.e. ballistically propagating structures in various observables as, for instance, in the heat fluxes ${ }^{27-30}$. The latter shall be considered in the following. As can be seen in Fig. 6, such structures are indeed present in nonlocal, collisionless GENE simulations of adiabatic-electron (ae) ITG turbulence using CBC-like parameters and they appear to be independent on whether a gradient or flux-driven mode is chosen. With these two simulations and a data base of further gradient-driven global simulations at hand, we can draw the following conclusions. Firstly, the avalanches appear to be governed by the underlying zonal flow dynamics since, e.g. the avalanche direction is linked to the sign of the $E \times B$ shearing rate and the radial extent limited to those regions with the same sign. In numbers, the propagation speed of the heat flux avalanches is of the order of $\rho^{*} c_{s}$ and the radial extent exhibits a mesoscale, i.e. about $20-40 \rho_{i}$, length scale. Both findings do not contradict a gyro-Bohm scaling, i.e. avalanches should also be visible in local simulations. Corresponding simulation results for CBC-like parameters are shown in Fig. 7. Clearly, avalanche-like structures can be observed for ITG turbulence on this microscopic $\left(\rho^{*} \rightarrow 0\right)$ scale, as well. However, considering kinetic electrons yields less pronounced structures which could be related to a weaker zonal flow activity in this case. The avalanches in any case exhibit no overall preference for the propagation direction averaged over the simulation domain which can be attributed to the periodic boundary conditions and the constant temperature and density profiles.

In line with the statements in Ref. 29, the avalanche propagation velocity is again close to $2 \rho_{i} / R_{0} c_{s}$ and corresponding radial correlation lengths being extracted are very similar. Fig. 7 furthermore displays heat fluxes for different types of modes as (low- $k$ ) temperature gradient driven trapped electron modes (TEMs) and electron temperature gradient driven 

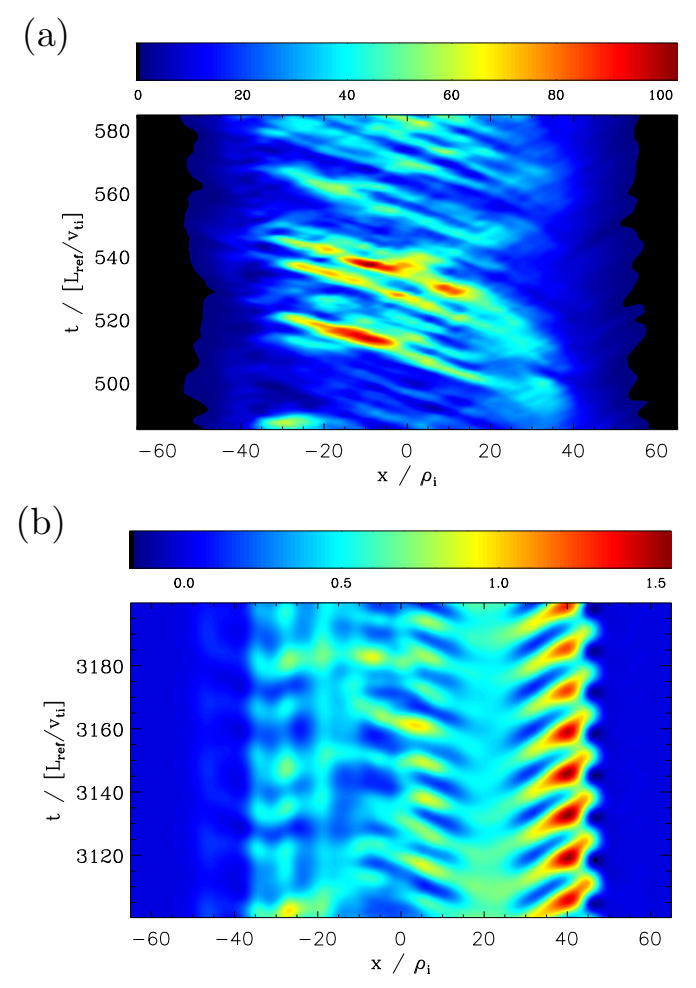

FIG. 6. (Color online) Flux surface averaged heat flux in units of $Q_{\mathrm{gB}}=p_{0} c_{s} \rho_{i}^{2} / R_{0}^{2}$ multiplied by the normalized flux surface area vs. time and radial coordinate. Plot (a) shows the result of a gradient driven simulation using the Krook type heat source while (b) contains the result of a "flux-driven" (by the localized physical heat source) simulation. Both simulations have been performed at $\rho^{*}=1 / 140$.

(ETG) high- $k$ modes. Here, avalanche structure are not visible at all, which is again in line with a weak zonal flow activity in these cases.

Since, in general, strong variations e.g. in the q-profile may affect particle orbits and wave-particle decorrelation physics, further (global) investigations are required to determine the parameters space where the above findings still hold. Additionally, externally triggered cold or hot pulses might exhibit a different behavior.

\section{APPLICATION TO A TCV TRANSPORT BARRIER}

Beyond benchmarks and investigations in artificial set-ups like the circular concentric equilibrium, the global GENE version has been run with realistic parameters and the actual MHD equilibrium taken from TCV discharge \#29866 (see Ref. 31) where an electron internal transport barrier (eITB) has been fully developed, see Fig. 8. As the initial profiles in Fig. 9 

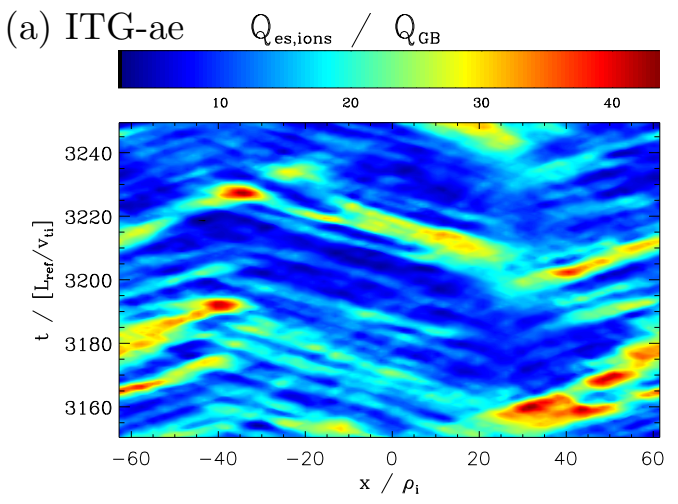

(c) ETG-ai Qes,electrons $/ Q_{G B}$

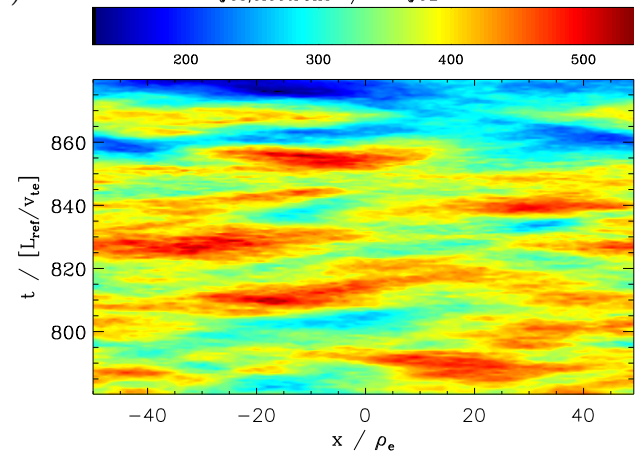

(b) ITG-ke $\quad Q_{\text {es,ions }} / Q_{\text {св }}$

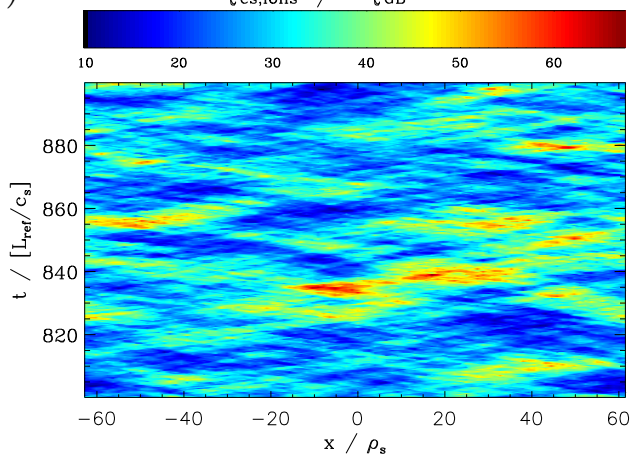

(d) TEM Q Q $\quad$ es,ions $/ Q_{\text {GB }}$

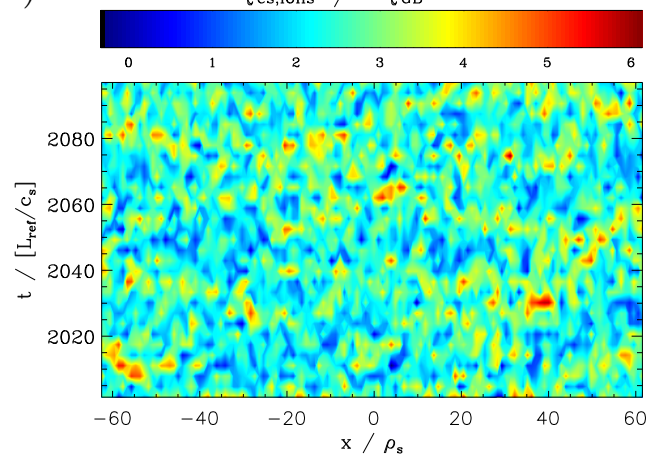

FIG. 7. (Color online) Flux surface averaged electrostatic heat fluxes for local GENE simulations. While avalanche-like structures can clearly be identified in the ITG driven cases (a) and (b), they are absent in (c) ETG driven turbulence with adiabatic ions (ai) and (d) temperature-driven TEM.

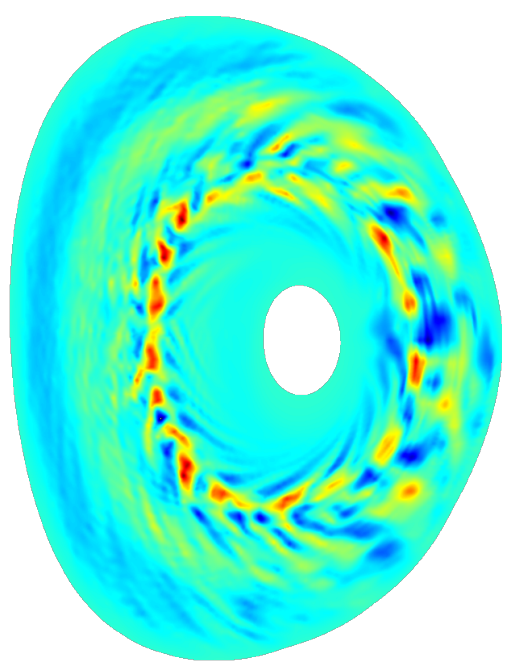

FIG. 8. (Color online) Cross section in the $R-Z$ plane of the electrostatic potential taken from a gyrokinetic simulation for TCV discharge \#29866.

indicate, it is characterized by very steep electron temperature and density gradients. The 


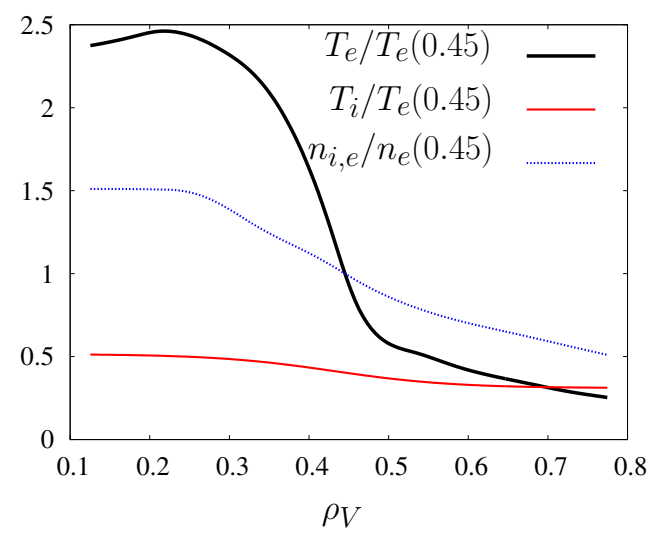

FIG. 9. (Color online) Initial temperature and density profiles for the simulation of TCV discharge \#29866 being normalized to the electron values at $\rho_{V}=\left(V / V_{\text {separatrix }}\right)^{1 / 2}=0.45$.

ion temperature profile is estimated to be approximately 4.5 times smaller than the electron temperature in the core but almost equal when approaching the edge. First simulations using the Krook-type heat and particle sources, electromagnetic effects and collisions but neglecting impurities, equilibrium $E \times B$ shear flow and considering a reduced mass ratio $m_{i} / m_{e}=400$ indeed recover a dominant electron heat flux. Given the dominant (electron) driving terms, most transport contributions can be attributed to TEM as has also been observed in linear global simulations ${ }^{32}$ as well as nonlinear local simulations ${ }^{33}$. The high- $k$ fraction, however, turns out to be non-negligible as the spectrum does not completely fall off at the highest currently resolved wave numbers. This - most likely, electron temperature gradient (ETG) driven - high- $k$ activity in transport barriers had already been suggested based on local simulations in the past, see e.g. Ref. 34. Considering the equilibrium $E \times B$ shear flow may exhibit a further increase of this fraction as the corresponding turbulence reduction mechanism tends to be most efficient at large wave lengths. However, at this point it should be mentioned that strong shear flows have not been observed in these TCV discharges, anyway, as there has been no additional source of momentum. Furthermore, the strength of the eITB is related to the ohmic current density which is typically not associated to $E \times B$ shearing ${ }^{35}$. Provided that future simulations including, e.g. impurities, confirm a significant high- $k$ activity, a full understanding of eITBs would require multi-scale simulations covering ion- and electron scales self-consistently. Naturally, such an approach would not just challenge the software but also the current hardware being at hand. Hence, it would represent one of the applications on present and future petascale systems. 


\section{CONCLUSIONS}

By considering radial temperature and density profiles as well as variations in the magnetic equilibrium, GENE - a grid based gyrokinetic turbulence code - has been significantly extended. However, the code still uses a field aligned coordinate system which allows for an efficient distribution of the simulation grid points. Both code versions, the local and the global one, have been compared and the local limit, i.e. a convergence of the global code towards the local result with decreasing $\rho^{*}$, has been confirmed. Hence, a gyro-Bohm transport scaling could be assumed for large fusion devices as, for instance, ITER. However, linear investigations as well as nonlinear results being derived from the codes OrB5 and GenE furthermore exhibit a significant dependence on the profile shape or the effective driving region, respectively. Significant deviations from the gyro-Bohm scaling are thus suggested in the presence of steep profiles or peaked gradient profiles as they occur, e.g. in internal transport barriers, even for small $\rho^{*}$ values.

A nonlocal effect possibly breaking the aforementioned scaling are so-called avalanches. These ballistically propagating structures have been identified and investigated via contours of the heat flux for a variety of different set-ups. In the absence of external pulses, they seem to be qualitatively independent on the type of operation, i.e. whether a local or a gradientor flux-driven global simulation has been chosen. However, due to a close relation to the zonal flow shearing rate they are mainly present in ITG driven turbulence and absent in temperature-driven TEM or ETG driven turbulence as zonal flows are less important in the latter cases.

Finally, first applications to discharges, here in the tokamak TCV, have been discussed which recover the dominant electron heat flux in eITBs while giving evidence that high- $k$ transport contributions might turn out to be non-negligible.

\section{ACKNOWLEDGMENTS}

This work was partly supported by the Swiss National Science Foundation. The simulations have been carried out on the Pleiades2 cluster at the EPFL, the RZG facilities in Garching, the HPC-FF computer and within the PRACE framework on the high performance computing resource (Tier-0) JUGENE at the Jülich Forschungszentrum, Germany. 


\section{REFERENCES}

${ }^{1}$ A. J. Brizard and T. S. Hahm, Rev. Mod. Phys 79, 421 (2007).

${ }^{2}$ X. Garbet, Y. Idomura, L. Villard, and T. H. Watanabe, Nuclear Fusion 50, 043002 (2010).

${ }^{3}$ K. V. Roberts and J. B. Taylor, Physics of Fluids 8, 315 (1965).

${ }^{4}$ S. C. Cowley, R. M. Kulsrud, and R. Sudan, Physics of Fluids B 3, 2767 (1991).

${ }^{5}$ M. A. Beer, S. C. Cowley, and G. W. Hammett, Phys. Plasmas 2, 2687 (1995).

${ }^{6}$ F. Jenko, W. Dorland, M. Kotschenreuther, and B. N. Rogers, Phys. Plasmas 7, 1904 (2000).

${ }^{7}$ T. Dannert and F. Jenko, Phys. Plasmas 12, 072309 (2005).

${ }^{8}$ F. Merz, Gyrokinetic Simulation of Multimode Plasma Turbulence, Ph.D. thesis, Westfälische Wilhelms-Universität Münster (2008).

${ }^{9}$ T. Görler, X. Lapillonne, S. Brunner, T. Dannert, F. Jenko, F. Merz, and D. Told, J. Comput. Phys.(2010), submitted.

${ }^{10}$ S. Jolliet, A. Bottino, P. Angelino, R. Hatzky, T. Tran, B. McMillan, O. Sauter, K. Appert, Y. Idomura, and L. Villard, Comput. Phys. Commun. 177, 409 (2007).

${ }^{11}$ B. F. McMillan, S. Jolliet, T. M. Tran, L. Villard, A. Bottino, and P. Angelino, Phys. Plasmas 15, 052308 (2008).

${ }^{12} \mathrm{X}$. Lapillonne, Microturbulence in electron internal transport barriers in the TCV Tokamak and global effects, Ph.D. thesis, École Polytechnique Fédérale de Lausanne (2010).

${ }^{13}$ T. Görler, Multiscale Effects in Plasma Microturbulence, Ph.D. thesis, Universität Ulm (2009).

${ }^{14}$ J. Candy, R. E. Waltz, S. E. Parker, and Y. Chen, Phys. Plasmas 13, 074501 (2006).

${ }^{15}$ Y. Idomura, M. Ida, S. Tokuda, and L. Villard, J. Comput. Phys. 226, 244 (2007).

${ }^{16}$ S. Jolliet, B. F. McMillan, T. Vernay, L. Villard, R. Hatzky, A. Bottino, and P. Angelino, Phys. Plasmas 16, 072309 (2009).

${ }^{17}$ M. J. Pueschel, T. Dannert, and F. Jenko, Comput. Phys. Comm. 181, 1428 (Aug. 2010).

${ }^{18}$ Y. Sarazin, V. Grandgirard, J. Abiteboul, S. Allfrey, X. Garbet, P. Ghendrih, G. Latu, A. Strugarek, and G. Dif-Pradalier, Nucl. Fusion 50, 054004 (2010).

${ }^{19}$ M. Fivaz, S. Brunner, G. de Ridder, O. Sauter, T. M. Tran, J. Vaclavik, L. Villard, and K. Appert, Comput. Phys. Commun. 111, 27 (1998).

${ }^{20}$ X. Lapillonne, B. F. McMillan, T. Görler, S. Brunner, T. Dannert, F. Jenko, F. Merz, and 
L. Villard, Phys. Plasmas(2010), accepted for publication.

${ }^{21}$ A. M. Dimits, G. Bateman, M. A. Beer, B. I. Cohen, W. Dorland, G. W. Hammett, C. Kim, J. E. Kinsey, M. Kotschenreuther, A. H. Kritz, L. L. Lao, J. Mandrekas, W. M. Nevins, S. E. Parker, A. J. Redd, D. E. Shumaker, R. Sydora, and J. Weiland, Phys. Plasmas 7, $969(2000)$.

${ }^{22}$ X. Lapillonne, S. Brunner, T. Dannert, S. Jolliet, A. Marinoni, L. Villard, T. Görler, F. Jenko, and F. Merz, Phys. Plasmas 16, 032308 (2009).

${ }^{23}$ B. F. McMillan, X. Lapillonne, S. Brunner, L. Villard, S. Jolliet, A. Bottino, T. Görler, and F. Jenko, Phys. Rev. Lett. 105, 155001 (2010).

${ }^{24}$ Z. Lin, S. Ethier, T. S. Hahm, and W. M. Tang, Phys. Rev. Lett. 88, 195004 (2002).

${ }^{25}$ J. Candy, R. E. Waltz, and W. Dorland, Phys. Plasmas 11, L25 (2004).

${ }^{26}$ L. Villard, A. Bottino, S. Brunner, A. Casati, J. Chowdhury, T. Dannert, R. Ganesh, X. Garbet, T. Görler, V. Grandgirard, R. Hatzky, Y. Idomura, F. Jenko, S. Jolliet, S. Khosh Aghdam, X. Lapillonne, G. Latu, B. F. McMillan, F. Merz, Y. Sarazin, T. M. Tran, and T. Vernay, Plasma Phys. Controlled Fusion 52, 124038 (2010).

${ }^{27}$ Y. Sarazin, X. Garbet, P. Ghendrih, and S. Benkadda, Phys. Plasmas 7, 1085 (2000).

${ }^{28}$ J. Candy and R. E. Waltz, J. Comput. Phys. 186, 545 (2003).

${ }^{29}$ B. F. McMillan, S. Jolliet, T. M. Tran, L. Villard, A. Bottino, and P. Angelino, Phys. Plasmas 16, 022310 (2009).

${ }^{30}$ G. Dif-Pradalier, P. H. Diamond, V. Grandgirard, Y. Sarazin, J. Abiteboul, X. Garbet, P. Ghendrih, A. Strugarek, S. Ku, and C. S. Chang, Phys. Rev. E 82, 025401 (2010).

${ }^{31}$ C. Zucca, O. Sauter, E. Asp, S. Coda, E. Fable, T. P. Goodman, and M. A. Henderson, Plasma Phys. Controlled Fusion 51, 015002 (2009).

${ }^{32}$ A. Bottino, O. Sauter, Y. Camenen, and E. Fable, Plasma Phys. Controlled Fusion 48, $215(2006)$.

${ }^{33}$ X. Lapillonne, B. F. McMillan, T. Görler, S. Brunner, T. Dannert, F. Jenko, F. Merz, and L. Villard, Plasma Phys. Controlled Fusion(2010), accepted for publication.

${ }^{34}$ F. Jenko, D. Told, P. Xanthopoulos, F. Merz, and L. D. Horton, Phys. Plasmas 16, 055901 (2009).

${ }^{35}$ O. Sauter, S. Coda, T. P. Goodman, M. A. Henderson, R. Behn, A. Bottino, E. Fable, A. Martynov, P. Nikkola, and C. Zucca, Phys. Rev. Lett. 94, 105002 (2005). 